\title{
Imagen multimodal en Epiteliopatía Pigmentosa Placoide Multifocal Posterior Aguda (APMPPE)
}

\author{
Pablo Vicente Muñoz-Ramon 1., *, Ana Isabel Fernández 2 , Francisco José Muñoz Negrete ${ }^{3}$, \\ Baram Bodaghi ${ }^{4}$ \\ 1 Servicio de Oftalmología, Hospital Universitario Ramon y Cajal, Madrid \\ 2 Servicio de Oftalmología, Hospital Universitario Pitié-Salpétrière \\ 3 Jefe de servicio de Oftalmología, Catedrático de oftalmología, Hospital Universitario Ramón y Cajal; \\ Departamento de Cirugía, Ciencias Médicas y Sociales, Universidad de Alcalá \\ 4 Jefe de servicio de Oftalmología, Catedrático de Oftalmología, Hospital Universitario Pitié-Salpétrière; \\ Facultad de Medicina, Sorbonne Université. \\ * Autor correspondencia: pablomuram@gmail.com \\ DOI: https://doi.org/10.37536/RIECS.2021.6.2.286
}

Recibido: 16/10/2021; Aceptado: 8/11/2021; Publicado: 30/11/2021

Resumen: Presentamos el caso de un varón de 28 años que consultó por pérdida de visión bilateral en el que el examen de fondo de ojo mostró lesiones compatibles con una epiteliopatía placoide multifocal posterior aguda asociada a una inflamación granulomatosa aguda de la cámara anterior. El presente caso sirve para ilustrar el proceso diagnóstico por imagen multimodal a nivel oftalmológico y el despistaje de posibles enfermedades asociadas o síndromes mascarada a nivel sistémico. Asimismo, la presentación del caso se acompaña de una revisión bibliográfica sobre la epiteliopatía en placas.

Palabras Clave: APMPPE, Síndromes de puntos blancos, Uveítis.

Abstract: We present the case of a 28-year-old man who consulted for bilateral vision loss in which the fundus examination revealed lesions consistent with acute posterior multifocal placoid pigment epitheliopathy associated with acute granulomatous inflammation of the anterior chamber. This case illustrates the multimodal diagnostic imaging process as well as the screening strategy for possible associated systemic diseases or masquerade syndromes. Likewise, the presentation of the case is accompanied by a bibliographic review on acute posterior multifocal placoid pigment epithelipathy.

Key words: APMPPE, White dot syndromes, Uveítis.

\section{Introducción}

La epiteliopatía pigmentosa placoide multifocal posterior aguda (APMPPE, por sus siglas en inglés), también conocida como "epiteliopatía en placas", es una enfermedad del complejo coriocapilar-EPR-retina externa que cursa con pérdida de visión indolora y afecta preferentemente a adultos jóvenes [1]. Aunque tradicionalmente la epiteliopatía en placas se ha considerado una enfermedad autolimitada hoy en día se sabe que puede recurrir y causar una pérdida de visión permanente. Adicionalmente, desde que fuera descrita por Gass en 1968, distintas series de casos sugieren la posible asociación de la misma con determinadas enfermedades sistémicas. Entre ellas figuran la infección por mycobacterium tuberculosis, la sarcoidosis, la sífilis y vasculitis a nivel del sistema nervioso central [2].

El adecuado conocimiento de esta enfermedad tiene por tanto una doble vertiente: por una parte es necesario hacer un diagnóstico diferencial oftalmológico correcto que permita excluir coroidopatías más agresivas (como la coroiditis ampiginosa) en las que el tratamiento con bolos de 
corticoides intravenosos está indicado sin dilación; por otra, está indicado un estudio sistémico adecuado con el fin de descartar comorbilidades asociadas y tratarlas en caso pertinente.

Presentamos el caso de un varón de 28 años con una APMPPE bilateral asociada a una uveítis granulomatosa que ilustra tanto la importancia de la imagen multimodal para el correcto diagnóstico de esta enfermedad como la sistemática a seguir para el estudio de posibles asociaciones a nivel sistémico.

\section{Material y Métodos}

Presentamos un caso clínico de una epiteliopatia placoide multifocal posterior aguda acompañado de una revisión bibliográfica narrativa. Para ello realizamos una búsqueda en septiembre de 2021 tanto en PubMed como en Google Scholar con las siguientes palabras clave: "acute posterior multifocal placoid pigment epitheliopathy", "APMPPE", "placoide epitheliopathy". Todos los artículos encontrados en español, inglés o francés fueron revisados en base a su abstract y aquellos considerados de mayor relevancia por los autores fueron incluidos para la revisión final.

\section{Resultados}

\subsection{Presentación del caso}

Presentamos el caso de un paciente varón de 28 años que consultó por pérdida de agudeza visual por el ojo izquierdo de unos 5 días de evolución. El paciente negaba cualquier antecedente oftalmológico o sistémico de interés así como contacto con animales (incluídas mascotas), tabaquismo, consumo de alcohol u otros tóxicos, contacto estrecho con pacientes con tuberculosis, contactos sexuales de riesgo o actividades al aire libre en zonas boscosas. Así mismo la anamnesis y la exploración por órganos y sistemas descartó signos sugerentes de patología respiratoria, digestiva, cutánea o articular.

A la exploración oftalmológica la agudeza visual con la mejor correción en el momento de la presentación 0,9 para su ojo derecho y 0,5 para su ojo izquierdo y la presión intraocular de $12 \mathrm{mmHg}$ para ambos ojos. La exploración en lámpara de hendidura reveló numerosos precipitados retroqueráticos de distribución estrellada en ambos ojos, un tyndall de 2+ y numerosos nódulos de Koeppe en el reborde iridiano de ambos ojos, todo ello compatible con una uveítis granulomatosa. En el fondo de ojo, por su parte, se objetivaron numerosas lesiones sobreelevadas, placoides, de 1 diámetro de disco aproximadamente, de color amarillento y bordes mal definidos localizadas en polo posterior y nasales a la papila del nervio óptico, tal y como se muestra en la figura 1.

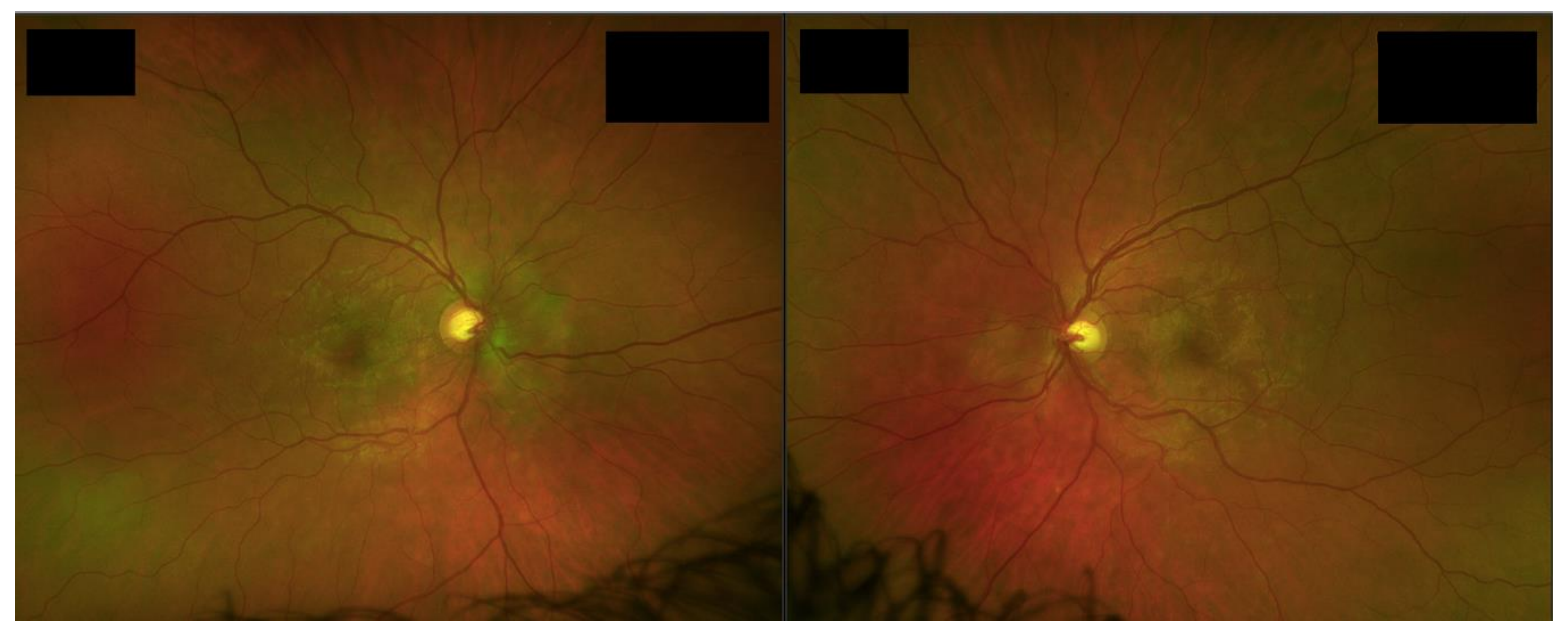

Figura 1 Aspecto del fondo de ojo a la presentación del paciente. Se observan múltiples lesiones placoides amarillentas mal definidas en polo posterior y nasales a la papila del nervio óptico 
Antes estas lesiones se consideró un amplio diagnóstico diferencial que incluyó todo el espectro de síndromes de puntos blancos, enfermedad de Birdshot y sarcoidosis ocular. Con el objetivo de precisar el diagnóstico se llevó a cabo un estudio de imagen multimodal consistente en tomografía de coherencia óptica (OCT), autofluorescencia del fondo y estudio angiográfico del ojo tanto con fluoresceína sódica como con verde de indocianina. En estas pruebas se observó lo siguiente:

1. OCT: lesiones hiperreflectivas a nivel de la retina externa y zona de elipsoides, con disrupción de los límites entre las distintas capas de la retina externa, en la misma localización que las lesiones placoides observadas en el fondo de ojo (Fig. 2)

2. Autofluorescencia del fondo: patrón moteado con una alternancia de áreas de hipo e hiperautofluorescencia que sugieren la existencia de zonas de sufrimiento del EPR con zonas de atrofia (Fig. 3).

3. Angiografía con fluoresceína: lesiones hipoautofluorescentes en tiempos precoces que se tornan hiperautofluorescentes en fase venosa tardía, compatible con una lesión isquémica a nivel de la coroides (Fig. 4).

4. Angiografía con verde de indocianina: reveló lesiones hipocianescentes tanto en tiempos precoces como en tiempos tardíos, de nuevo compatibles con isquemia coroidea (Fig. 5). Asimismo, la ausencia de lesiones hipocianescentes en tiempos precoces con captación de contraste en tiempos tardíos permitió descartar la presencia de granulomas coroideas asociados, aspecto de gran relevancia diagnóstica dada la asociación de una inflamación granulomatosa en cármara anterior.

5. Campo visual Humphrey 24-2 y 10-2: no se objetivaron defectos en el ojo derecho. Por su parte el ojo izquierdo mostró numerosos escotomas centrales tal y como muestra la figura 6.
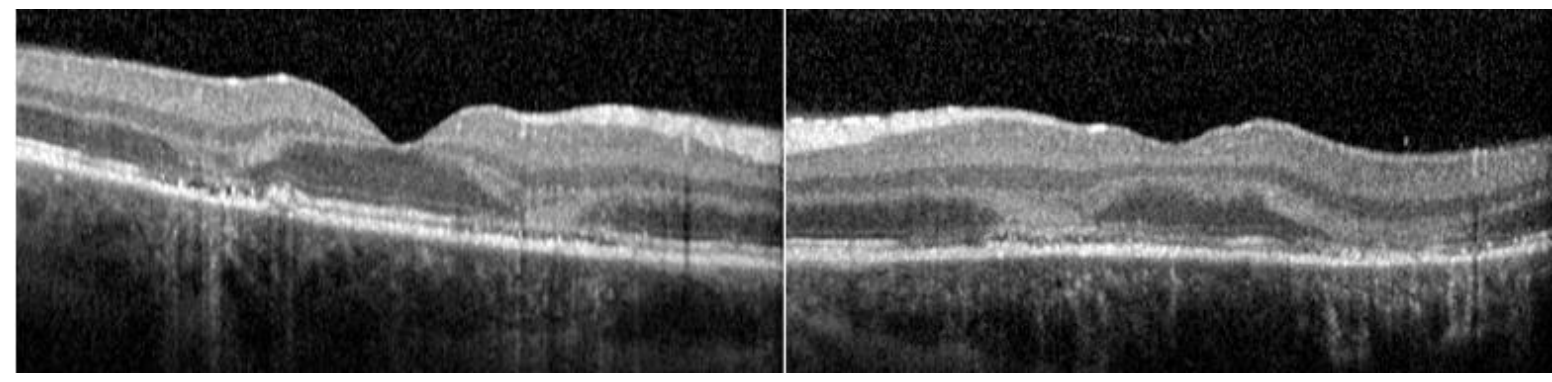

Figura 2 OCT de las lesiones en el momento agudo: lesiones hiperreflectivas con disrupción de la retina externa coincidentes con las lesiones placoides observadas en el fondo de ojo 


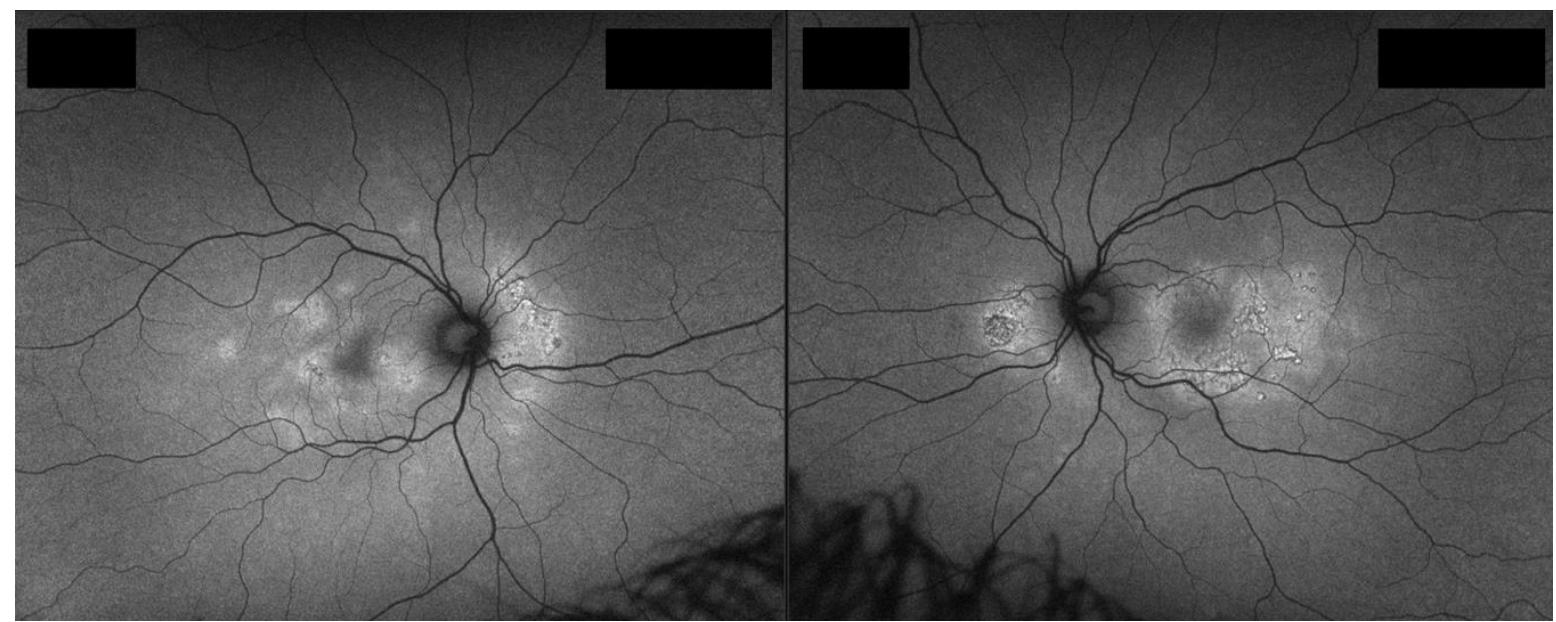

Figura 3 Autofluorescencia del fondo de ojo que muestra la alternancia de zonas hipo e hiperautofluorescentes en las zonas de las lesiones funduscópicas

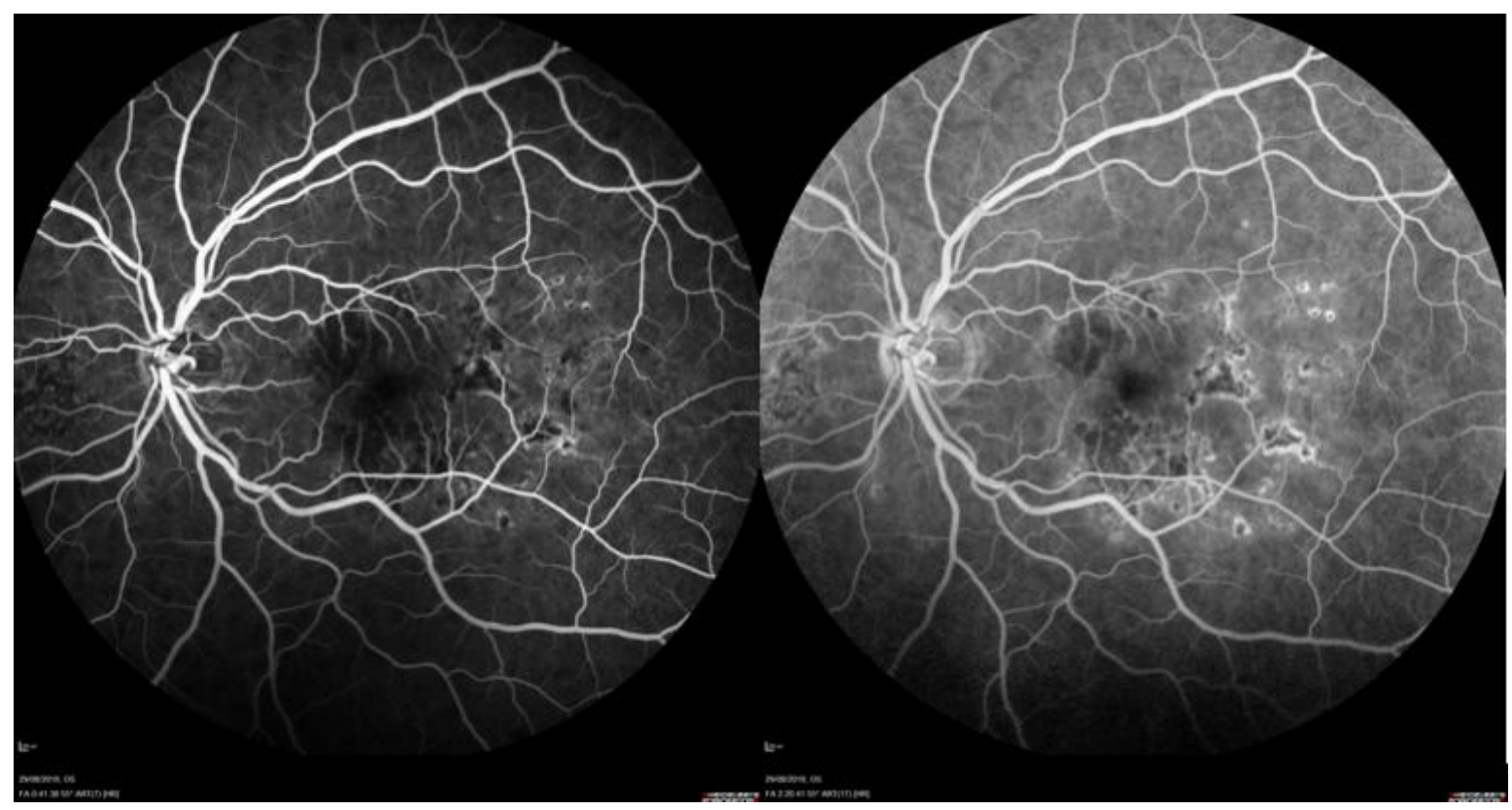

Figura 4 Angiografía con fluoresceína del ojo izquierdo del paciente en la fase aguda. A la izquierda, la fase venosa precoz (43 segundos) muestra lesiones hipofluorescentes. A la derecha, las fases tardías (8 minutos) muestran lesiones hiperfluorescentes de relleno centrípeto, compatibles con lesiones coroideas. La angiografía fluoresceínica del ojo adelfo mostró un patrón idéntico. 


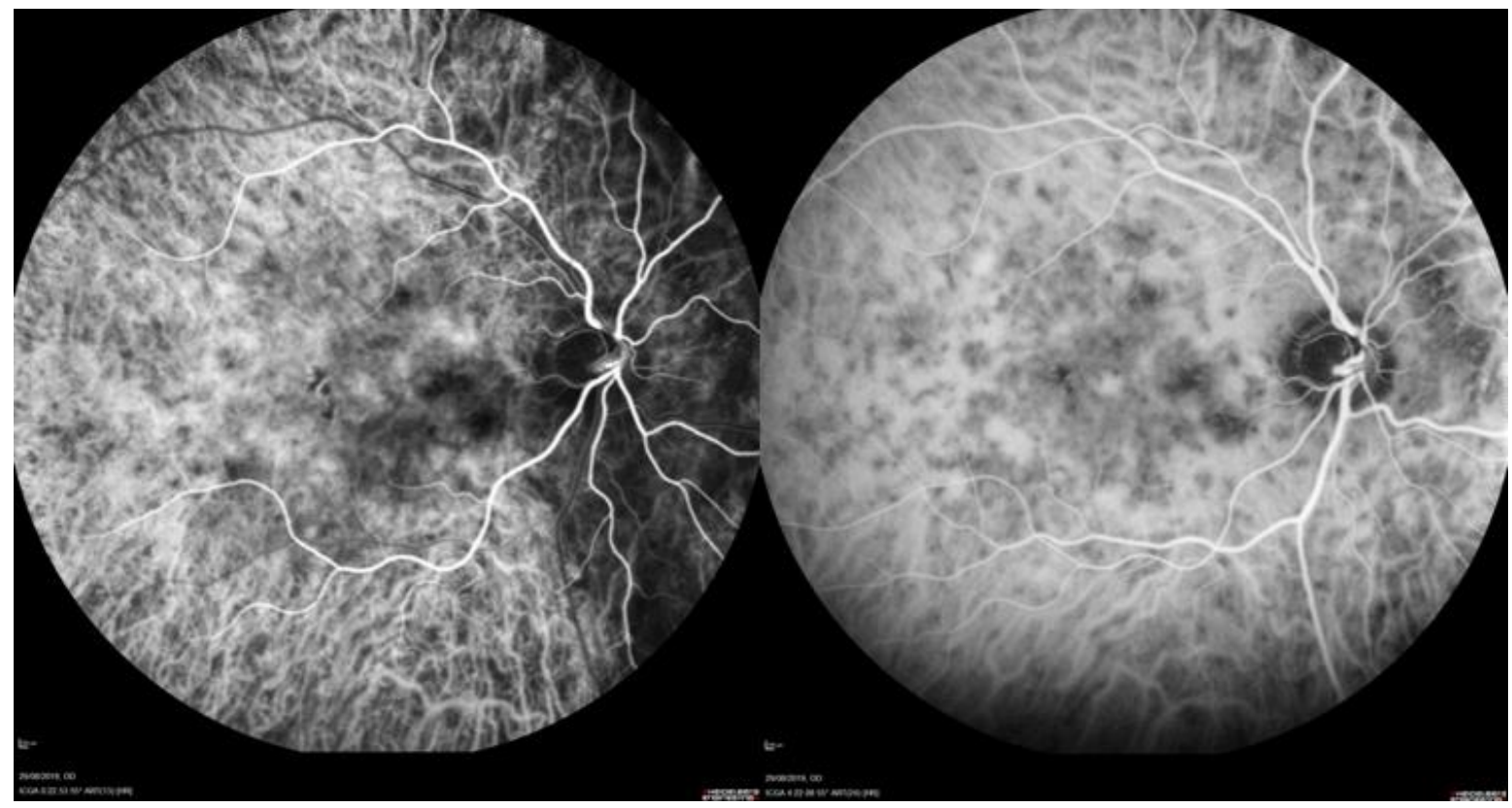

Figura 5 Angiografía con verde de indocianina del ojo derecho del paciente en el momento agudo. Tanto los tiempos precoces (izquierda) como tardíos (derecha) muestran lesiones hipocianescentes. El examen del ojo adelfo mostró lesiones idénticas.
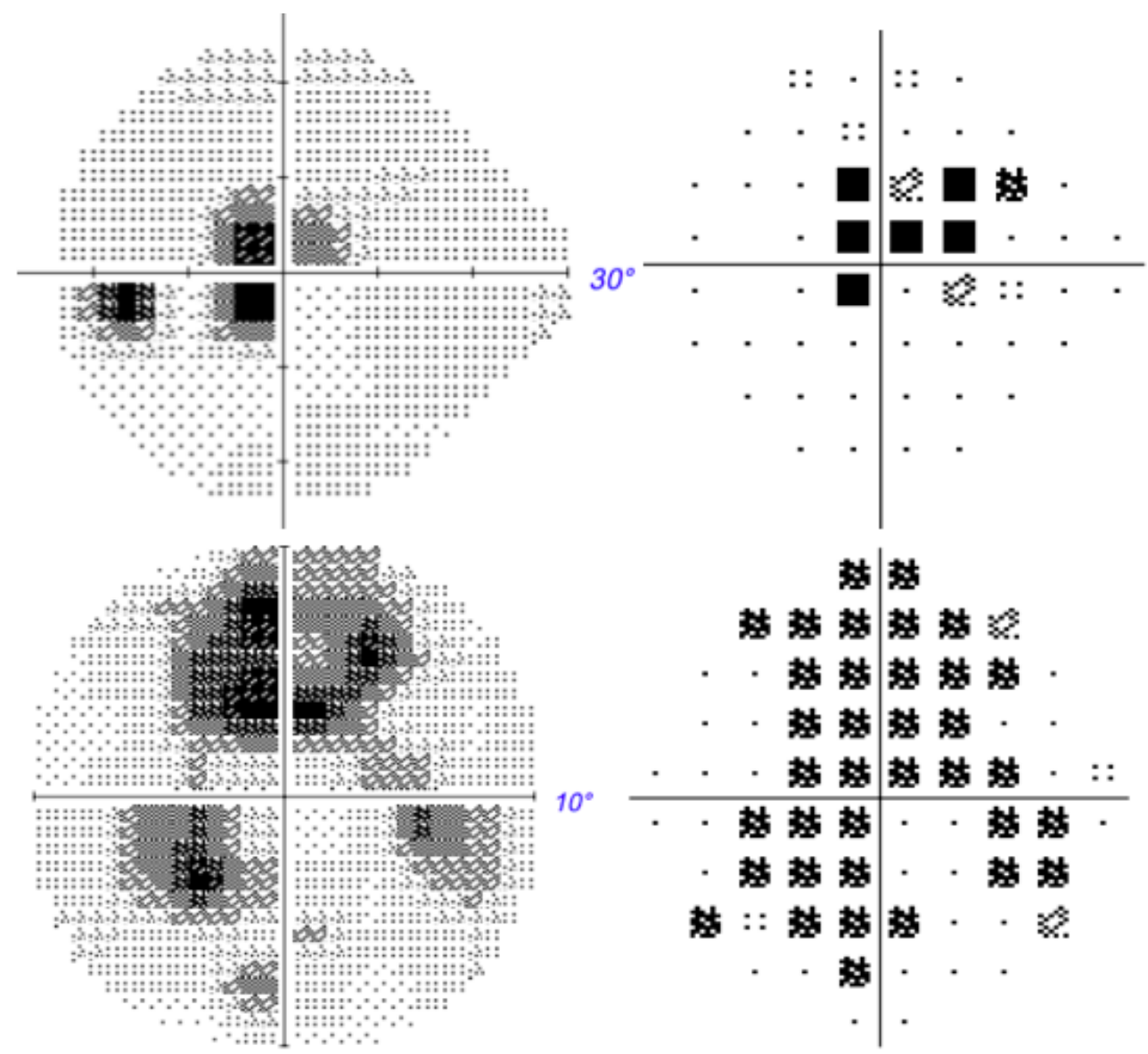

Figura 6 Mapa de grises y mapa de desviación en patrón del campo visual Humphrey 24-2 (arriba) y 10-2 (abajo) a la presentación del paciente. Nótese la correalación de los diversos escotomas centrales con las placas objetivadas en el fondo de ojo y la disrupción de los segmentos externos en la OCT 
Los resultados del estudio de imagen permitieron establecer el diangóstico de presunción de APMPEE en el contexto de una panuveítis granulomatosa. Por este motivo se tomó la decisión de ingresar al paciente para estudio etiológico y seguimiento.

\subsection{Estudio sistémico y tratamiento}

Ante las caracterísitcas de este caso se procedió a realizar un estudio sistémico centrado en el despistaje de posibles enfermedades asociadas a una uveítis anterior granulomatosa acompañada de una epiteliopatía en placas posterior. Las entidades incluidas en este despistaje y las pruebas solicitadas a tal efecto son las que se detallan a continuación:

- $\quad$ Tipaje de HLA-A29* y HLA-B27*, en el primer caso por su fuerte asociación con la Enfermedad de Birdshot y en el segundo por su asociación con la uveítis anterior aguda idiopática.

- Sarcoidosis, solicitándose por tanto placa de tórax y niveles séricos de enzima convertidora de angiotensina (ECA) y lisozima.

- Serología de aquellas enfermedades infecciosas cuya asociación con uveítis anterior granulomatosa o cuyas manifestaciones en el polo posterior están bien establecidas: sífilis, VIH, VHB, VHC, enfermedad de Lyme, toxoplasmosis así como quatiferon para mycobacterium tuberculosis.

Todo el estudio sistémico resultó negativo, clasificándose por tanto la entidad que presentaba el paciente como uveítis anterior granulomatosa acompañada de epiteliopatía en placas. Respecto a la conducta terapéutica, una vez descartadas posibles causas infecciosas se decidió iniciar un pauta de dexametasona tópica al 0,1\%, comenzando con 6 gotas al día y decreciendo la dosis en 1 gota cada semana.

\subsection{Evolución}

El paciente acudió a sus revisiones periódicas y cumplió de forma correcta el tratamiento indicado. A las 6 semanas del diagnóstico, se comprobó una resolución completa de la inflamación de la cámara anterior y una mejoría de las lesiones del polo posterior, con aplanamiento de las mismas y comienzo de evolución hacia la atrofia y la hiperpigmentación.

La figura 7 muestra el aspecto del fondo de ojo del ojo izquierdo tras un año de seguimiento y la mejoría de los escotomas centrales en el campo visual en comparación con los exámenes realizados al diagnóstico (ver figura 6).

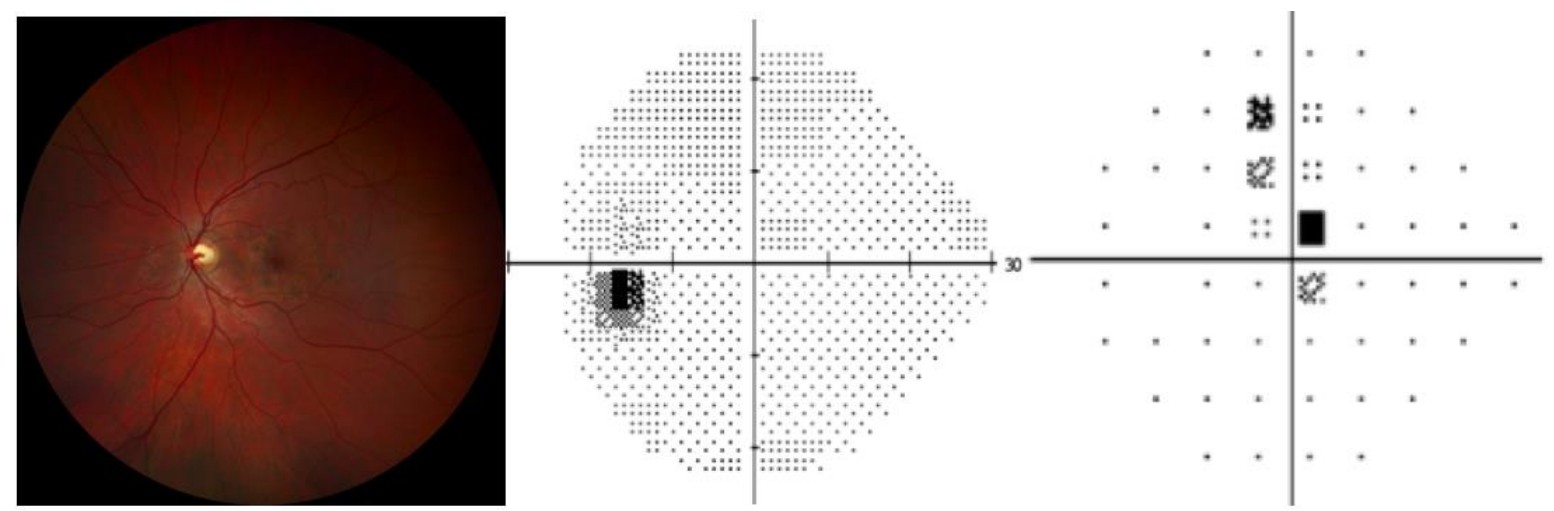

Figura 7 Retinografía del ojo izquierdo, mapa de grises y mapa de desviación en patrón del ojo izquierdo del paciente 1 año tras el diagnóstico. Nótese la evolución hacia la atrofia e hiperpigmentación de las lesiones del polo posterior y la mejoría de los escotomas centrales en el campo visual 
Tras un seguimiento de más de 2 años no se han producido recidivas de su enfermedad ni a nivel anterior ni posterior. Por otra parte, tampoco se han producido signos o síntomas a nivel sistémico que hayan llevado a plantear la necesidad de repetir el estudio sitémico. El paciente continúa con revisiones periódicas cada 6 meses con diagnóstico de un único episodio de uvéitis anterior granulomatosa asociada a epiteliopatía en placas.

\section{Discusión}

La epiteliopatía placoide posterior multifocal aguda es una enfermedad de etiología indeterminada. En la actualidad hay controversia sobre si se trata de una enfermedad inmunomediada o de un proceso isquémico. No obstante, existe cierto consenso sobre que el lugar donde ocurre la enfermedad de forma primaria se encuentra a nivel de la coriocapilar, produciéndose una disrupción de los segmentos externos de los fotorreceptores y del EPR de forma secundaria [1]. La enfermedad afecta predominantemente a pacientes de entre 20 y 40 años sin precilección por sexos [2].

Clínicamente la APMPPE se caracteriza por la aparición de lesiones placoides sobreelevadas, amarillentas, de bordes mal definidos y localización posterior al ecuador. Los pacientes se suelen quejar de pérdida de visión bilateral e indolora de instauración brusca, que puede ir acompañadas de fotopsias [1,3]. Algunos pacientes son capaces de precisar que la pérdida de visión es en realidad debida a la percepción de escotomas centrales, tal y como puede objetivarse en el campo visual.

El presente caso sirve para ilustar la conducta a seguir ante un paciente con lesiones placoides en el polo posterior. Estos pacientes deben ser aproximados desde una doble vertiente: en primer lugar, desde el punto de vista oftalmológico se debe plantear un diagnóstico diferencial amplio que incluya, entre otros, las enfermedades del espectro de los síndromes de puntos blancos. En este sentido el estudio del ojo con imagen multimodal será fundamental para distinguir unas entidades de otras. En segundo lugar, debe tenerse presente que diversas enfermedades sistémicas (como la tuberculosis o la sarcoidosis) pueden estar asociadas a algunos síndromes de puntos blancos a la vez que otras (como la sífilis o la enfermedad de Lyme) pueden dar manifestaciones oftalmológicas cuyo aspecto clínico se puede solapar con el de los síndromes de puntos blancos. El despistaje de enfermedades sistémicas en un paciente con APMPEE se explica por tanto por un doble motivo: la posible asociación de algunas enfermedades con esta entidad y la posibilidad de que las lesiones oftalmológicas sean una manifestación de una enfermedad subyacente multisistémica y no una enfermedad puramente oftalmológica [4].

Otro aspecto a destacar a la hora de aproximar el estudio sistémico del paciente con APMPPE que es este debe considerarse un proceso dinámico a lo largo del cual el paciente puede desarrollar una enfermedad sistémica no diagnosticada en el momento de hacer el estudio etiológico inicial. Por tanto, los pacientes con antecedentes de APMPEE deben ser vigilados periódicamente y ante la aparición de signos o síntomas sugerentes de algunas de las enfermedades nombradas con anterioridad no se debe durar en repetir las pruebas pertinentes. De esta forma, muchas uveítis idiopáticas son reclasificadas años después de su debut como uveítis asociadas a una entidad concreta.

Como se ha comentado antes el estudio sistémico debe combinarse con un estudio de imagen multimodal a nivel oftalmológico que permita la caracterización precisa de la enfermedad y la valoración del nivel de actividad. Así, los pacientes con sospecha de APMPPE o de otro síndrome de puntos blancos deben de ser estudiados con la siguientes pruebas [5]: retinografía, OCT, campo visual, autofluorescencia del fondo de ojo y angiografía fluoresceínica y con verde de indocianina. Los hallazgos característicos de la APMPPE en estas pruebas son los que se muestran en la Tabla I. 
Tabla I Hallazgos característicos de APMPPE en imagen multimodal

\begin{tabular}{|c|c|c|c|c|}
\hline FONDO & OCT & AUTOFLÚOR & AGF & ICG \\
\hline $\begin{array}{c}\text { Lesiones } \\
\text { multifocales } \\
\text { de } \\
\text { morfología } \\
\text { placoide y } \\
\text { color } \\
\text { amarillento, } \\
\text { levemente } \\
\text { sobreelevad } \\
\text { a y de } \\
\text { bordes mal } \\
\text { definidos. }\end{array}$ & $\begin{array}{c}\text { En estadíos } \\
\text { precoces } \\
\text { hiperreflectivida } \\
\text { d de las capas } \\
\text { externas de la } \\
\text { retina. En } \\
\text { estadíos } \\
\text { cicatriciales } \\
\text { disrrución de } \\
\text { segmentos } \\
\text { externos de } \\
\text { fotorreceptores y } \\
\text { atrofia de EPR. }\end{array}$ & $\begin{array}{c}\text { Lesiones } \\
\text { hipoautofluorescentes } \\
\text { en fase aguda. } \\
\text { En fase cicatricial } \\
\text { patrón moteado con } \\
\text { alternancia de hipo e } \\
\text { hiperautofluorescenci } \\
\text { a. }\end{array}$ & $\begin{array}{c}\text { Lesiones } \\
\text { hipofluorescentes } \\
\text { en tiempos } \\
\text { precoces que se } \\
\text { tornan } \\
\text { hiperfluorescente } \\
\text { s en tiempos } \\
\text { tardíos. }\end{array}$ & $\begin{array}{c}\text { Lesiones } \\
\text { hipocianescente } \\
\text { s en todas las } \\
\text { fases, más } \\
\text { numerosas que } \\
\text { las observadas } \\
\text { en fondo de ojo } \\
\text { o en AGF. }\end{array}$ \\
\hline
\end{tabular}

Desde el punto de vista terapéutico la mayoría de los autores recomienda observar a los pacientes con APMPPE, ya que esta enfermedad suele evolucionar espontáneamente hacia la curación sin necesidad de tratamiento [1]. En aquellos casos recidivados o con una afectación extensa del polo posterior se puede iniciar tratamiento con corticoides sistémicos en pauta descendente. En nuestro caso se instauró tratamiento con corticoides tópicos debido a la existencia de una inflamación granulomatosa asociada en el cámara anterior que se resolvió sin complicaciones con un único ciclo de dexametasona al 0,1\% descendente.

Finalmente, un último aspecto a destacar del caso presentado es la asociación de la APMPPE con una inflamación granulomatosa en la cámara anterior. Tradicionalmente la APMPPE se ha descrito como una enfermedad puramente del polo posterior del ojo sin presencia de inflamación en la cámara anterior. No obstante, existe al menos otros dos reportes en la literatura de casos en los que una APMPPE se asoció a una inflamación anterior granulomatosa [6,7], el primero de ellos idiopático y el segundo atribuido a la administración de una dosis de vacuna contra la gripe por su asociación temporal. En este sentido hay que tener en cuenta que el conocimiento que se tiene hoy en día de las enfermedades inflamatorias del ojo continúa siendo limitado. Presumiblemente muchas de ellas comparten procesos patogénicos similares que podrían explicar la existencia de cierto grado de solapamiento entre las mismas.

\section{Conclusiones}

La APMPPE es una enfermedad de etiología desconocida que afecta a adultos jóvenes. Su importancia reside en la necesidad de hacer un correcto estudio oftalmológico que permita confirmar el diagnóstico y excluir otras entidades más agresivas que requieren tratamiento antimicrobiano o inmunosupresor sin dilación. Adicionalmente es necesario llevar a cabo un estudio sistémico amplio con el fin de descartar enfermedades asociadas o patologías que pueden cursar con lesiones similares a la APMPPE en el fondo de ojo.

Agradecimientos: los autores no han contado con ninguna fuente de financiación para el presente trabajo.

Contribución de los autores: P.V.M.R fue el encargado de llevar a cabo la revisión bibliográfica y la redacción del documento. A.I.F, F.J.M.N y BB concibieron el formato del caso clínico, contribuyeron a la revisión bibliográfica y fueron los responsables de la supervisión y revisión del documento final.

Conflictos de Intereses: los autores no declaran conflicto de intereses. 


\section{Abreviaturas}

Las siguientes abreviaturas son usadas en este manuscrito:

APMPPE: Acute Posterior Multifocal Placoid Pigment Epitheliopathy (Epiteliopatía Placoide Posterior Multifocal Aguda).

EPR: Epitelio Pigmentario de la Retina.

HLA: Human Leukocyte Antigen (Antígeno Leucocitario Humano)

VIH: Virus de la Inmunodeficiencia Humana

VHB: Virus de la Hepatitis B

VHC: Virus de la Hepatitis C

OCT: Optical Coherence Tomography (Tomografía de Coherencia Óptica)

AGF: Angiografía Fluoresceínica

ICG: Indocyanine Green (Verde de Indocianina)

\section{Referencias Bibliográficas}

1. Testi I, Vermeirsch S, Pavesio C. Acute posterior multifocal placoid pigment epitheliopathy (APMPPE). Journal of Ophthalmic Inflammation and Infection (2021) 11:31.

2. Jones NP. Acute posterior multifocal placoid pigment epitheliopathy. Br J Ophtalmol 79 (4):384-389

3. Gass JDM. Acute posterior multifocal placoid pigment epitheliopathy. Arch Ophthalmol 80(2):177-185.

4. Russel J F, Pichi F, Scott N L, Hartley M J, Bell D, Agarwal A et al. Masqueraders of multiple evanescent white dot syndrome (MEWDS). Int Ophthalmol. 2020 Mar;40(3): 627-638.

5. Raven M L, Ringeisen A L, Yonekawa Y, Stem M S, Faia L J, Gottlieb JL. Multi-modal imaging and anatomic classification of the white dot syndromes. Int J Retin Vitr (2017) 3:12.

6. Alvi N P, Fishman G A. Granulomatous anterior uveitis presenting with acute posterior multifocal placoid pigment epitheliopathy. Doc Ophthalmol. 1995; 89 (4): 347-53

7. Gonome T, Suzuki Y, Metoki T, Takahashi S, Nakazawa M. Acute posterior multifocal placoid pigment epitheliopathy and granulomatous uveitis following influenza vaccination. Am J Ophthalmol Case Rep. 2016 Aug 31; 4:60-63.

(C) 2021 por los autores; Esta obra está sujeta a la licencia de Reconocimiento 4.0 Internacional de Creative Commons. Para ver una copia de esta licencia, visite http://creativecommons.org/licenses/by-nc-nd/4.0/. 